\title{
Electrophysiologic and antiarrhythmic actions of nadolol: Acute ischemia in the presence of previous myocardial infarction
}

\begin{abstract}
The actions of the $\beta$-adrenergic receptor antagonist, $\alpha, 1$-nadolol, were examined in anesthetized dogs subjected to circumflex coronary artery ligation in the presence of previous anterior myocardial infarction. With circumflex ligation, control dogs $(N=18)$ developed premature ventricular beats and ventricular tachycardia, followed by ventricular fibrillation $(N=16,89 \%)$. Immediate arrhythmias ( 2 to 5 minutes) were accompanied by activation delays and continuous diastolic electrical activity in acutely ischemic epicardial tissue. Delayed arrhythmias (6 to 12 minutes) were accompanied by delayed activation and continuous diastolic electrical activity in acutely ischemic mid-myocardium. Nadolol $(8 \mathrm{mg} / \mathbf{k g}$, intravenously) $(N=10)$ reduced ventricular arrhythmias during both phases of arrhythmia development and increased survival $(70 \%$, $p=0.001$ vs control). Nadolol failed to alter activation in acutely ischemic epicardium, but prevented beat-to-beat changes in epicardial and mid-myocardial activation. Atrial pacing of nadolol-treated animals at heart rates comparable with those of the control group reversed the beneficial effects of nadolol on the development of ventricular arrhythmias and ventricular fibrillation ( $70 \% ; p=0.07$ vs nadolol; $p=0.21$ vs control, respectively). The beneficial effects of nadolol could not be attributed to reduced epicardial delays, but were associated with the suppression of beat-to-beat conduction abnormalities that preceded ventricular fibrillation. (AM HEART J 1988;116:1223.)
\end{abstract}

Eugene Patterson, PhD, and Benedict R. Lucchesi, PhD, MD. Ann Arbor, Mich.

The ligation of a major coronary artery in the dog produces rapid alterations in the electrical properties of the myocardium served by the vessel. Delayed electrical activation is observed within ischemic tissue, which reflects a decrease in conduction velocity or a change in the activation pattern of the ischemic myocardium..$^{1-4}$ Premature ventricular beats, ventricular tachycardia, and less frequently ventricular fibrillation are sequelae of acute ischemia. If a major coronary artery is ligated in the presence of previous myocardial infarction within the distribution of a second coronary artery, both the incidence and severity of premature ventricular beats, ventricular tachycardia, and ventricular fibrillation are increased over that observed with the ligation of a single major coronary vessel. ${ }^{s, b}$ The

From the Department of Pharmacology, Iniversity of Michigan Medical School.

Supported by a grant from the Heart, Lung, and Blood Institute, National Institutes of Health. The research was performed while Dr. Patterson was a John G. Searle Fellow in Clinical Pharmacology.

Received for puhlication Feb. 22. 1988; accepted July 1, 1988.

Reprint requests: Eugene Patterson, PhD. Research Service 151-F, Veterans Administration Medical Center, $921 \mathrm{NE} 13$ th St., Oklahoma City, OK 73104. electrophysiologic basis for the increased arrhythmias with ischemia in the presence of previous infarction is not well understood. Although increased epicardial activation delays within acutely ischemic myocardium are present, ${ }^{6}$ the increase in activation delays and arrhythmogenesis has been at least partially attributed to an increase in sympathetic nervous system tone or a compensatory sinus tachycardia elicited by an acute ischemic event. ${ }^{5.7}$

Experimental interventions capable of reducing the incidence of ventricular fibrillation when acute ischemia is superimposed on previous distant myocardial infarction include $\beta$-adrenergic receptor blockade with $d, 1$-nadolol ${ }^{7,8}$ and left stellate ganglionectomy. ${ }^{5}$ The interventions blunt the heart rate response to an acute ischemic event in the conscious dog and presumably may also block other potentially detrimental electrophysiologic consequences of increased activity of the sypathetic nervous system in acutely ischemic tissue. The present study was undertaken to determine the possible electrophysiologic bases for the antiarrhythmic and antifibrillatory effects of $\beta$-adrenergic receptor blockade by $d, 1$-nadolol in the anesthetized dog subjected to left circumflex coronary artery ligation in the presence of previous acute myocardial infarction. 


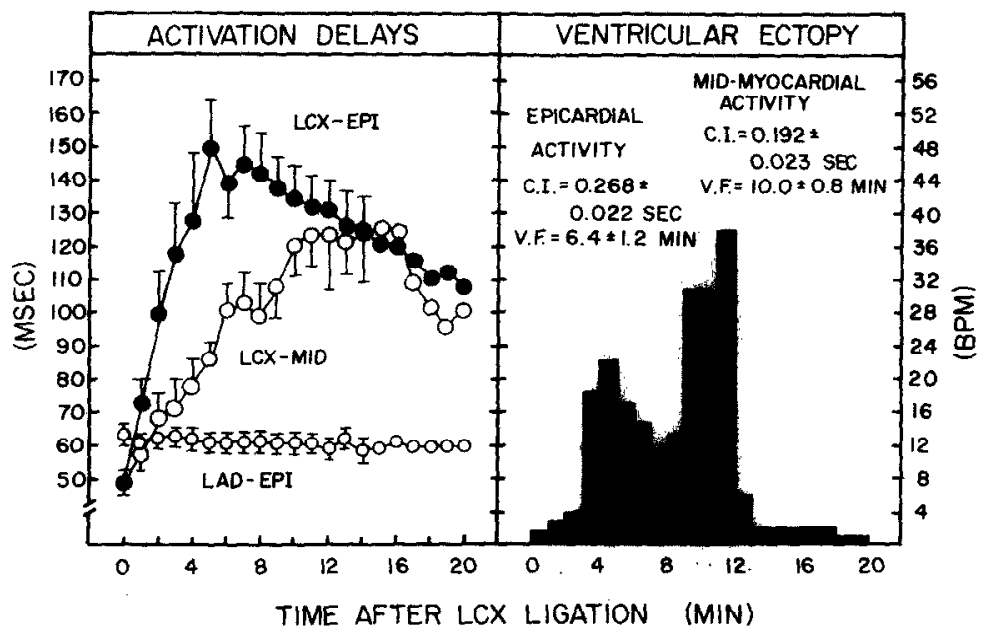

Fig. 1. Activation delays and ventricular ectopy in the control treatment group subjected to left circumflex ( $L C X)$ coronary artery occlusion in the presence of previous anterior myocardial infarction. Conduction delays were measured in left circumflex epicardium ( $L C X-E P I)$, left anterior descending epicardium (LAD-EPI), and left circumflex mid-myocardium (LCX-MID) before and at 1-minute intervals after left circumflex coronary artery ligation. Two separate phases of arrhythmia development were observed. The first phase occurred within the first 6 minutes after coronary artery ligation in conjunction with delayed activation and continuous diastolic electrical activity measured on the epicardial surface of the left circumflex coronary artery. The second or delayed phase of arrhythmia development occurred after 8 minutes and occurred simultaneously with the development of increased activation delays and continuous diastolic electrical activity within mid-myocardium of the left circumflex distribution. CI, Coupling interval of premature ventricular beat.

\section{METHODS}

Animal preparation. Male mongrel dogs were anesthetized with intravenous sodium pentobarbital $(30 \mathrm{mg} / \mathrm{kg})$. A cuffed endotracheal tube was inserted, and the animal was ventilated with a Harvard respirator. With aseptic technique, the left common carotid artery and left external jugular vein were isolated and indwelling catheters were inserted. A left thoracotomy was performed in the fifth intercostal space, and the heart was suspended in a pericardial cradle. The left anterior descending coronary artery was isolated at the tip of the left atrial appendage. A 19-gauge needle was placed parallel to the artery, and a suture was passed around both the artery and the needle. The suture was tied and the needle withdrawn, which created a critical stenosis. Previous experiments ${ }^{9}$ have demonstrated the ability of the stenosis to reduce the hyperemia observed with the reinstitution of coronary flow after temporary occlusion of the vessel. The stenosis does not reduce the resting blood flow through the vessel, but reduces the incidence of ventricular fibrillation on reperfusion and prevents hemorrhagic infarction. ${ }^{9}$ The artery was then occluded with a snare formed by passing a loop of silicone rubber tubing through a section of blunted polyethylene tubing. After 2 hours, flow was restored through the stenosed artery. The chest was closed, and the animals were allowed to recover from surgery. A mpicillin, $\bar{i} \mathrm{iug} / \mathrm{kg}$, was administered at surgery and on the first postoperative day.

Experimental procedure. On day 5 after myocardial infarction, the animals were anesthetized with $30 \mathrm{mg} / \mathrm{kg}$ of intravenous sodium pentoharbital. A cuffed endotracheal tube was inserted, and the animals were ventilated with room air with a Harvard respirator. Arterial blood pressure was measured with a Statham blood pressure transducer. A left thoracotomy was performed in the fifth intercostal space, and the heart was suspected in a pericardial cradle. With a 22 -gauge hypodermic needle $(1.5$ inches in length), insulated silver wires $(0.05 \mathrm{~mm}$ diameter) with 2 to $3 \mathrm{~cm}$ exposed recording surfaces were implanted intramurally in the left circumflex coronary artery distribution, parallel to the first obtuse marginal branch. Composite bipolar recordings were obtained by summing five or six electrode pairs with an approximate 2 to $3 \mathrm{~mm}$ interelectrode distance. Bipolar composite electrodes were placed on the epicardial surface of the left ventricle. ${ }^{2}$ One electrode was placed over ischemically injured myocardium in the left anterior descending coronary artery distribution, and a second electrode was placed on the epicardial surface of the left circumflex coronary artery distribution. All composite electrodes were filtered at 50 and $500 \mathrm{~Hz}$. Activation delays were measured both before and at selected intervals after ligation of the left circumflex coronary artery. The measurement of the activation delays was performed directly fromin the display of a Tektronix Model 5111 storage oscilloscope. In some experiments an electrocatheter was advanced through the left carotid artery and advanced to the root of the aorta to record His-bundle activation. 


\section{MINUTES}

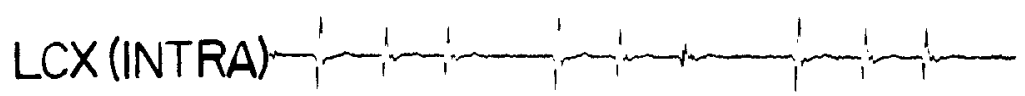

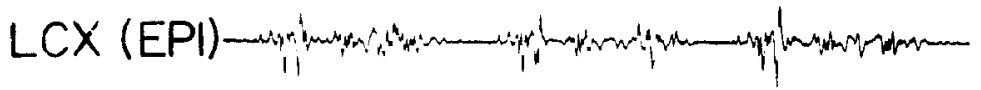

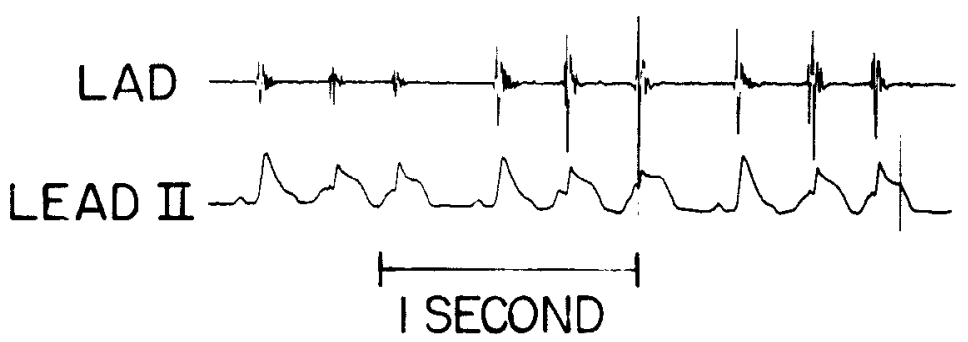

Fig. 2. Electrophysiologic recordings obtained 5 minutes after left circumflex coronary artery ligation. Composite electrode recordings were obtained from mid-myocardium within the left circumflex distribution ( $L C X[I N T R A])$ and from the epicardial surface of the left circumflex ( $L C X[E P I])$ and left anterior descending ( $L A D)$ distributions. A lead II ECG was recorded from the body surface. The recordings were obtained 5 minutes after ligation of the left circumflex coronary artery. Continuous electrical activity bridging diastole is present on the epicardial surface of the left circumflex distribution, which connects the sinus heart beats and all three ventricular couplets. The diastolic period is electrically quiescent in the remaining composite electrograms.

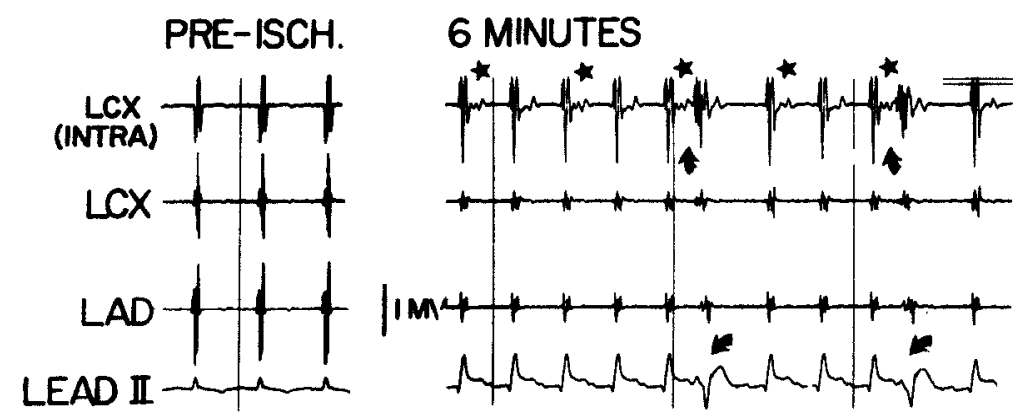

Fig. 3. Electrophysiologic recordings obtained 6 minutes after left circumflex coronary artery ligation. The electrical recordings are as described in Fig. 2. In the left hand panel, composite electrograms are shown during sinus rhythm, before left circumflex coronary artery ligation. Activation in each electrogram remains discrete and localized within the QRS interval of the surface ECG. In the right hand panel, electrical recordings obtained 6 minutes after left circumflex coronary artery ligation are shown. Delayed electrical activity is present in the composite electrograms recorded from both mid-myocardium and epicardium within the left circumflex coronary artery distribution. The delay is greater in mid-myocardium than on the epicardial surface. Local 2:1 conduction block is present in midmyocardium. Two single premature ventricular beats (arrows) are present and are associated with the prolonged duration electrogram of the 2:1 conduction block, as well as continuous mid-myocardial electrical activity bridging the diastolic interval between the sinus beat and the ventricular beat. The mid-diastolic interval remains quiescent in the remaining two composite electrograms.

Recordings were also obtained at $100 \mathrm{~mm} / \mathrm{sec}$ with an oscillographic recorder. Continuous records were obtained of blood pressure and a lead II ECG with a Grass Model 7 polygraph. The activation delay was defined as the interval between the $Q$ wave of the lead II ECG and the cessation of local electrogram activity. ${ }^{2,10}$ Nll measure ments are then mean of triplicate determinations obtained during sinus rhythm.

A 2-hour temporary occlusion of the left anterior descending artery followed by reperfusion through a critical stenosis was performed in 51 male mongrel dogs. Electrophysiologic studies were performed in 39 surviving 


\subsection{MINUTES}

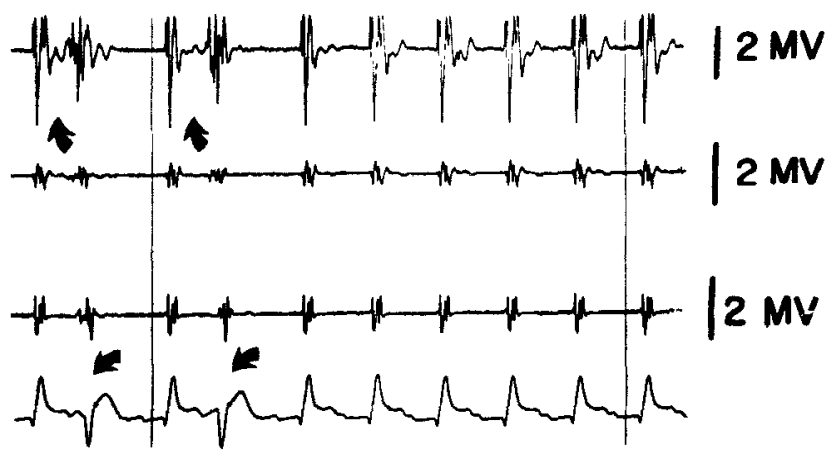

Fig. 4. Electrophysiologic recordings obtained 6.2 minutes after left circumflex coronary artery ligation. Fig. 4 is a continuation of the experiment described in Fig. 3. The recordings are as previously described. Premature ventricular beats (arrows) are accompanied by continuous diastolic electrical activity recorded in the mid-myocardial composite electrogram.

dogs on day 5 after myocardial infarction. The initial studies $(N=8)$ were performed with only saline solution pretreatment. The remaining 30 studies were performed after randomization of the experimental preparations to control (saline), nadolol, and nadolol + atrial pacing (200 $\left.\mathrm{min}^{-1}\right)$ treatment groups. Saline solution (10 ml) or nadolol $(8 \mathrm{mg} / \mathrm{kg}$ in $10 \mathrm{ml}$ of saline) was administered intravenously over a 10-minute period. All treatments were administered 15 minutes before circumflex coronary artery ligation proximal to the first obtuse marginal branch. In the nadolol + atrial pacing group, the left atrium was stimulated at a rate of $193 \pm 5$ beats $/ \mathrm{min}$ for the first 30 minutes of coronary artery ligation $(4 \mathrm{msec}$ stimuli, two times diastolic threshold). On completion of the experiment 6 hours after left circumflex coronary artery ligation or on the development of cardiac arrest, the heart was removed and rinsed in cold water. The heart was then cut into $5 \mathrm{~mm}$ thick slices parallel to the atrioventricular groove. The heart was stained for the presence of active intracellular dehydrogenases with $0.5 \%$ triphenyltetrazolium chloride in $0.01 \mathrm{~mol} / \mathrm{L}$ phosphate buffer, $\mathrm{pH}$ 7.4. Infarct size was determined gravimetrically and was expressed as a percentage of the total left ventricular mass.

Statistics. The data are expressed as the mean \pm the standard error of the mean. One-way analysis of variance was used to determine differences between treatment groups. Individual differences between groups were determined with Bonferoni's test. Fisher's exact test was used to determine differences in survival between treatment groups.

\section{RESULTS}

Ventricular arrhythmia development in the control (saline) treatment group. Within 1 minute after cir-
Table 1. Sinus heart rates in control, $d, 1$-nadolol, and $d, 1$-nadolol + pacing treatment groups

\begin{tabular}{rrrc}
\hline & Control & Nadolol & Nadolol + pacing \\
\hline Predrug & $145 \pm 16$ & $147 \pm 8$ & $142 \pm 3$ \\
Postdrug & $142 \pm 16$ & $106 \pm 8^{*}$ & $107 \pm 9^{*}$ \\
Post left circumflex ligation (min) & \\
2 & $188 \pm 10^{*}$ & $120 \pm 10^{* \dagger}$ & $193 \pm 5^{*}$ \\
5 & $190 \pm 12^{*}$ & $122 \pm 9^{* \dagger}$ & $194 \pm 4^{*}$ \\
10 & 186 & $124 \pm 12^{*}$ & $190 \pm 6^{*}$ \\
30 & 148 & $126 \pm 10^{*}$ & $188 \pm 9^{*}$ \\
\hline
\end{tabular}

${ }^{*} p<0.01$ vs predrug.

$\dagger p<0.05$ vs control and nadolol + pacing.

cumflex coronary artery occlusion, fractionation and delay of normally conducted beats were apparent in the composite electrograms recorded from the acutely ischemic circumflex coronary artery distribution. The delay progressively increased and reached a maximal value of $149 \pm 13 \mathrm{msec}$ on the epicardial surface of the circumflex distribution at 5 minutes. As the activation delay increased on the epicardial surface, the frequency and severity of ventricular arrhythmia also increased (Fig. 1). Single premature ventricular beats were first observed at $2.2 \pm 1.2$ minutes, with a mean epicardial delay of $138 \pm 12 \mathrm{msec}(N=18)$. Ventricular tachycardia was observed at $4.6 \pm 1.4$ minutes, at a mean epicardial delay of $156 \pm 16 \mathrm{msec}(N=18)$. In each instance, the ventricular arrhythmia was accompanied by the presence of continuous diastolic electrical activity on the epicardial surface of the circumflex coronary artery distribution. In 10 dogs, ventricular rhythms accompanied by continuous epicardial diastolic electrical activity in the circumflex coronary artery distribution ended in ventricular fibrillation (6.4 \pm 1.2 minutes) (Fig. 1). Ventricular fibrillation was preceded by beat-to-beat changes in the electrogram configuration recorded from epicardial surface of the left circumflex coronary artery distribution. The magnitude of the delays in the mid-myocardium during the immediate arrhythmia phase was always less than that observed on the epicardial surface, and continuous local electrical activity spanning diastole during ventricular arrhythmia was not observed in the acutely ischemic mid-myocardium.

Fig. 2 shows composite electrogram recordings from mid-myocardial (intramural), epicardial circumflex, and epicardial anterior descending sites. T'he electrogram recordings were obtained 5 minutes after circumflex coronary artery occlusion. Three episodes of ventricular couplets after a sinus heart beat are shown. In each example, the diastolic 


\subsection{MINUTES}

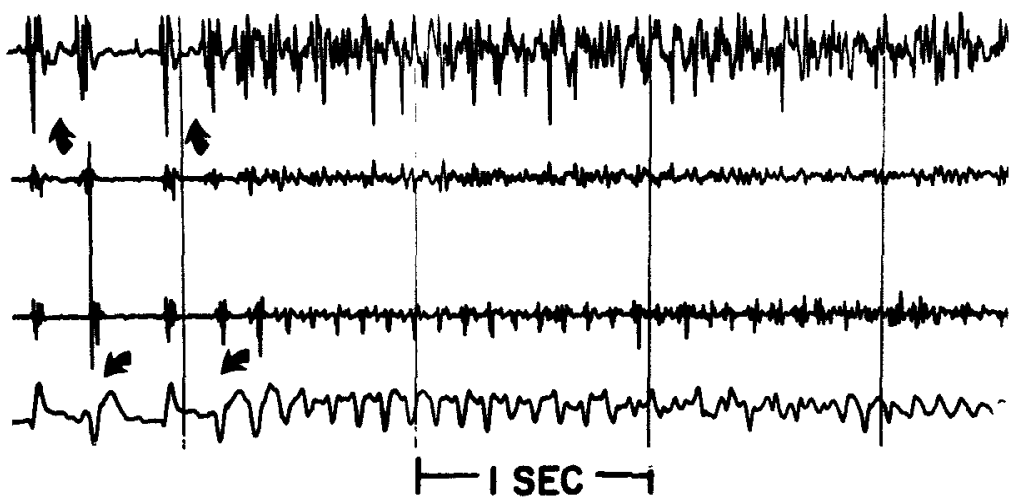

Fig. 5. Electrophysiologic recordings obtained 8.0 minutes after left circumflex coronary artery ligation. Fig. 5 is a continuation of the same experiment described in Figs. 3 and 4 . At 8.0 minutes after left circumflex coronary artery ligation, ventricular tachycardia degenerates to ventricular fibrillation. The first two beats of the tachycardia/fibrillation are accompanied by continuous electrical activity recorded in mid-myocardium only.

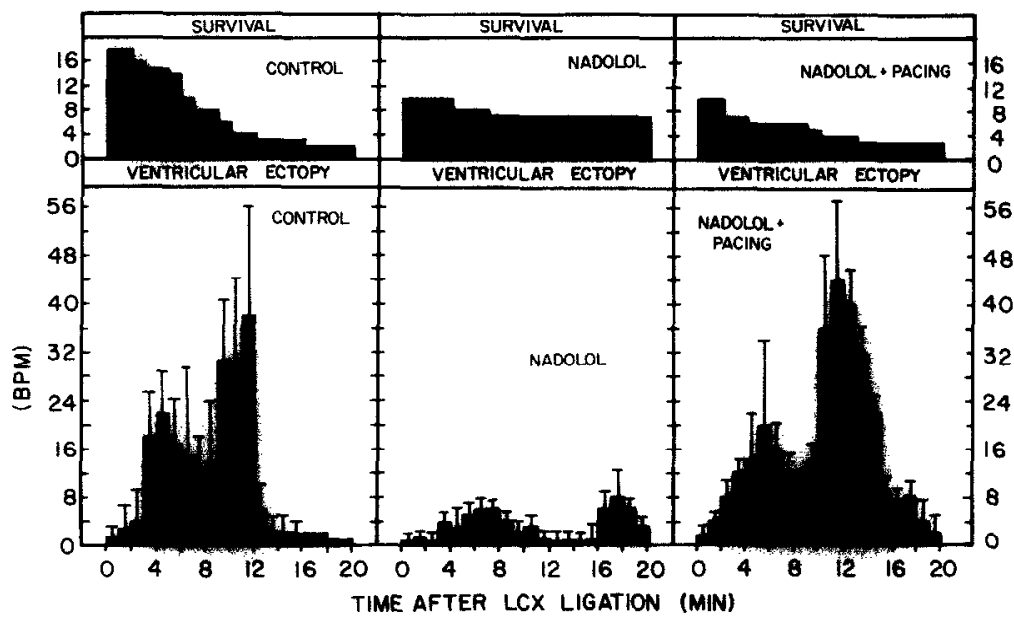

Fig. 6. Ventricular arrhythmias in control, nadolol, and nadolol + pacing treatment groups. Survival and the incidence of ventricular arrhythmia are graphed for control, $d, 1$-nadolol, and $d, 1$-nadolol + pacing treatment groups subjected to left circumflex coronary artery ligation in the presence of previous anterior myocardial infarction. Nadolol pretreatment reduced the incidence of ventricular arrhythmias and prolonged survival compared with the control treatment group. Atrial pacing reversed the beneficial effects of nadolol administration with no significant differences in survival or arrhythmias between the nadolol + pacing and control groups. (Nadolol treatment group. $t$ (time) $=2$ to 6 minutes, 10 to 12 minutes, $p<0.05$ vs control and nadolol + pacing group).

interval preceding the ventricular beat is spanned by continuous electrical activity in the epicardial composite electrogram recording from the circumflex distribution. Activation within the anterior descending epicardium and circumflex mid-myocardium remains discrete and localized to systole. In the six experiments that used His-bundle recordings, activation of the His-bundle occurred during the QRS complex.

The immediate phase of arrhythmia development was followed by a second, delayed phase of ventricular arrhythmia. The delayed phase was preceded by further increases in mid-myocardial activation delays within the left circumflex distribution. The duration of electrical activity in the mid-myocardial (intramural) zone frequently exceeded the delay observed on the epicardial surface (Fig. 1). The site of earliest electrical activation for the second arrhythmia phase was consistently observed in the mid-myocardium. A substantial number of the ven- 


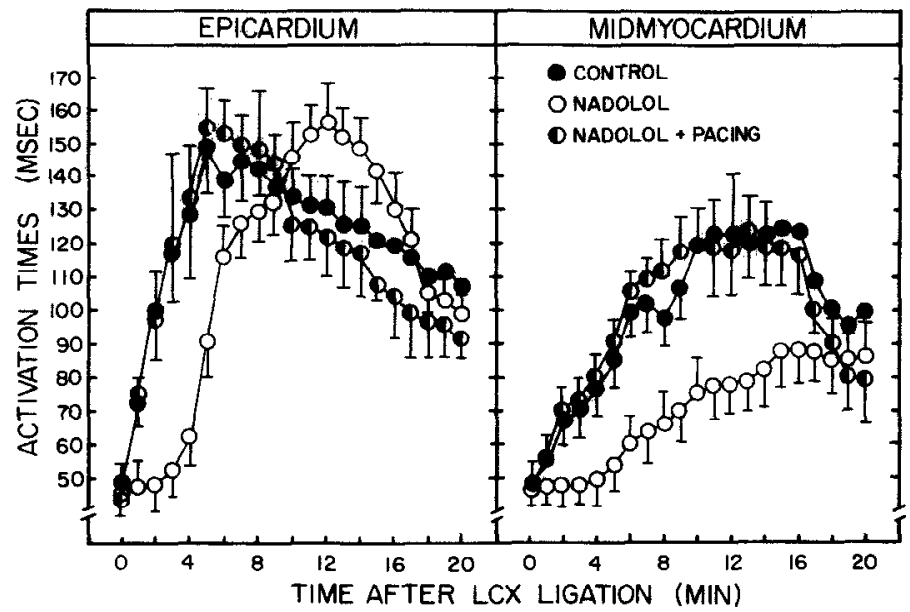

Fig. 7. Left circumflex epicardial and mid-myocardial conduction delays in control, nadolol, and nadolol + pacing treatment groups. Epicardial and mid-myocardial conduction delays are shown for the three treatment groups. Nadolol administration delayed but failed to reduce the maximal conduction delay present after left circumflex coronary artery ligation, with the maximal delay occurring at $12 \pm 3$ vs $5 \pm 2$ minutes. Nadolol treatment reduced the maximal delay measured in mid-myocardium $(86 \pm 12$ msec) when compared with control $(126 \pm 23 \mathrm{msec})$ and nadolol + pacing treatment groups $(138 \pm 25$ msec) $(p<0.01)$. (Nadolol treatment group, epicardium, $\mathrm{t}$ (time) $=2$ to 6 minutes, $p<0.05$ vs control and nadolol + pacing group; mid-myocardium, $t=2$ to 14 minutes, $p<0.05$ vs control and nadolol + pacing groups)

tricular ectopic beats occurring during this second phase at $9.0 \pm 1.0$ minute $(23 \% \pm 8 \%)$ was accompanied by continuous mid-myocardial diastolic electrical activity preceding the ventricular ectopic beat. The second arrhythmia phase produced ventricular tachycardia at $9.6 \pm 0.9$ minute. In six of eight remaining dogs, ventricular fibrillation occurred at $10.0 \pm 0.8$ minute after circumflex occlusion. Unlike the immediate phase of ventricular arrhythmia, which consisted of ventricular ectopic beats with morphologic findings of left bundle-branch block, the delayed phase arrhythmias were principally of a right bundle-branch block morphology and were more closely coupled with the sinus heart beat (192 \pm 23 vs $268 \pm 22 \mathrm{msec}, p<0.01$ ). His-bundle activation preceded the QRS complex. Representative recordings from the delayed phase of arrhythmia development are shown in Figs. 3 to 5 .

Nadolol treatment group. Nadolol $(8 \mathrm{mg} / \mathrm{kg}$ intravenously) was administered to $10 \mathrm{dogs}, 15$ minutes before circumflex coronary artery ligation. Nadolol administration was accompanied by a decrease in the sinus heart rate from $147 \pm 8$ to $106 \pm 8$ beats/ min. With circumflex coronary artery ligation, the heart rate increased to $120 \pm 10$ beats $/ \mathrm{min}$ $(\omega$ - v.ua) and was not further altered over the 6 -hour duration of the experiment (Table I). The heart rate response to circumflex coronary artery ligation in the nadolol group differed markedly from that observed in the control group. In the control group, circumflex coronary artery occlusion produced an immediate increase in the sinus heart rate from $142 \pm 6$ to $188 \pm 10$ beats/min (Table I). Sinus heart rates after nadolol treatment were significantly less than those observed in the control and nadolol + pacing groups (Table I).

Nadolol administration suppressed both immediate and delayed arrhythmia development after circumflex coronary artery ligation. The incidence of premature ventricular beats (Fig. 6) and the subsequent development of ventricular fibrillation ( $N=1, p=0.001$ ) were reduced compared with the control group. Seven of 10 nadolol-treated dogs survived 6 hours, with electromechanical dissociation as the cause of death in two animals ( $p=0.02 \mathrm{vs}$ saline solution, $p=0.08$ vs nadolol + pacing) (Fig. 6). However, nadolol did not alter the extent of epicardial activation delay present after circumflex ligation (Fig. 7), but increased the time for the development of maximal epicardial delay from $4.8 \pm 1.2$ to $12.2 \pm 3.4$ minutes $(p<0.05$ vs control and nadolol + pacing groups). Mid-myocardial activation delays during the first 16 minutes of circumflex occlusion were reduced significantly in the nadolol group $(p<0.05$ vs control and nadolol + pacing groups). No beat-to-beat alterations in the epicardial or mid-myocardial composite electrograms were observed after circumflex occlusion in eight of 10 nadolol-treated animals, which is in contrast with beat-to-beat changes in activation 
observed in control and nadolol + pacing groups $(17 / 18, p=0.0004 ; 9 / 10, p=0.003$, respectively). An example of a 2:1 conduction disorder in the acutely ischemic zone mid-myocardial composite electrogram of a saline-treated animal can be observed in Fig. 3.

Nadolol + atrial pacing treatment group. With intravenous nadolol $(8 \mathrm{mg} / \mathrm{kg})$, there was an immediate decrease in the sinus heart rate from $142 \pm 3$ to $107 \pm 9$ beats $/ \mathrm{min}(p<0.01)$. Beginning 15 seconds before circumflex artery occlusion, atrial pacing was used to maintain the heart rate at $193 \pm 5$ beats $/ \mathrm{min}$ (Table I). The paced heart rate in the nadolol + pacing group was not different from the spontaneous heart rates observed in the control group.

With circumflex coronary artery occlusion, there was immediate fractionation and delay in the composite electrograms recorded from acutely ischemic epicardium and mid-myocardium. Initially, the epicardial activation delays always exceeded those observed in the mid-myocardium. Accompanying circumflex epicardial electrogram fractionation and delay was the development of continuous diastolic electrical activity and ventricular ectopic beats at $2.4 \pm 1.3$ minutes (Figs. 6 and 7). Ventricular tachycardia was observed at $3.9 \pm 1.2$ minutes after circumflex ligation at an mean epicardial delay of $162 \pm 16 \mathrm{msec}$. There were no differences in the extent or timing of epicardial and mid-myocardial activation delays in the control and nadolol + pacing groups, and there were no differences in the incidence or timing of the two phases of arrhythmia development (Figs. 6 and 7). Four animals in the nadolol + atrial pacing group developed ventricular fibrillation in conjunction with ventricular ectopic beats, resulting from continuous diastolic electrical activity in acutely ischemic epicardium $(3.4 \pm 1.2$ minutes). The delayed phase of arrhythmia formation produced a recurrence of ventricular premature beats and ventricular tachycardia at $8.6 \pm 1.2$ minutes and $9.2 \pm 1.1$ minutes $(N=6)$, respectively, followed by ventricular fibrillation in three of six surviving animals (11.2 \pm 0.8 minutes). The delayed phase of ventricular ectopia and ventricular fibrillation was associated with delayed activation and diastolic electrical bridging in mid-myocardium but not in acutely ischemic epicardium.

Acute circumflex coronary artery ligation was associated with significant decreases in mean arterial pressure in all three treatment groups. There were no differences in mean arterial pressure between treatment groups throughout the experiment (Table II).

Myocardial infarct mass. Anterior descending infarct mass in saline solution, nadolol, and nadolol + pacing groups was $17 \% \pm 3 \%, 16 \% \pm 3 \%$,
Table II. Mean arterial pressures in control, $d, 1$-nadolol, and $d, 1$-nadolol + pacing treatment groups

\begin{tabular}{cclc}
\hline & Control & Nadolol & Nadolol + pacing \\
\hline Before drug & $112 \pm 11$ & $106 \pm 10$ & $113 \pm 9$ \\
After drug & $114 \pm 12$ & $101 \pm 9$ & $109 \pm 9$ \\
After left circumflex ligation & $(\mathrm{min})$ & \\
2 & $94 \pm 10^{*}$ & $88 \pm 12^{*}$ & $86 \pm 11^{*}$ \\
5 & $90 \pm 11^{*}$ & $86 \pm 10^{*}$ & $83 \pm 9^{*}$ \\
10 & 78 & $87 \pm 13^{*}$ & $91 \pm 8^{*}$ \\
30 & 77 & $87 \pm 12^{*}$ & $82 \pm 9^{*}$ \\
\hline
\end{tabular}

${ }^{*} p<0.05$ vs predrug.

and $16 \% \pm 4 \%$ of total left ventricular mass, respectively. Infarct mass in the circumflex coronary artery distribution in the seven surviving dogs of the nadolol treatment group was $21 \% \pm 4 \%$ of left ventricular mass.

\section{DISCUSSION}

Mechanisms for ventricular arrhythmia formation during the immediate phase of ventricular arrhythmia development (initial 8 to 10 minutes) after left clrcumflex coronary artery occlusion. During the initial 8 to 10 minutes after left circumflex coronary artery ligation, progressive increases in the duration of epicardial activation were recorded from the left circumflex coronary artery distribution. The mid-myocardial delays were always less than the recorded epicardial delays, presumably because of the slowed but normal transmural excitation pathway from endocardium to epicardium. Ventricular premature beats were invariably (1) preceded by delayed epicardial activation, which exceeded $150 \mathrm{msec}$ on the composite electrogram recorded from the left circumflex coronary artery distribution, (2) preceded by localized conduction disorders (i.e., 2:1 or Wenckebach-like conduction disturbances in acute ischemic epicardial electrograms), and (3) accompanied by continuous epicardial electrical activity bridging the diastolic interval from the preceding ventricular beat. Premature ventricular beats were not observed during the immediate phase of ventricular arrhythmia unless the epicardial delays exceeded $150 \mathrm{msec}$ and could presumably exceed the local recovery of myocardial excitability. There was no evidence for the active participation of previously injured anterior epicardium in acute arrhythmia formation. Electrical activity recorded from the anterior distribution remained discrete until rapid ventricular tachycardia ( $>300$ beats $/ \mathrm{min}$ ) had developed. The development of continuous electrical activity within the anterior epicardial electrogram was associated with the eventual degeneration of ventricular tachycardia to ventricular fibrillation. 
The mechanism for ventricular arrhythmia formation during the immediate ventricular arrhythmia phase closely parallels that previously described by Waldo and Kaiser, ${ }^{1}$ Williams et al., ${ }^{2}$ and Kaplinsky et $\mathrm{al}^{3}$ for acute anterior descending coronary artery ligation. These investigators have attributed the earliest phase of acute arrhythmia development to localized epicardial reentry within the ischemic zone. This conclusion was largely made on the basis of the demonstration of diastolic electrical bridging between an initiating and a "reentrant" ventricular beat. Other evidence suggesting local myocardial reentry as a mechanism for the immediate phase of ventricular arrhythmia consisted of (1) the appearance of local conduction block (that is, 2:1 conduction block, Wenckebach-like conduction, etc.) preceding ventricular arrhythmia and (2) the increases in epicardial conduction delays exceeding the approximate duration of recovery of excitability. An epicardial site for reentry is also consistent with the observation of His-bundle activation during and not preceding the QRS complex and morphologic findings of left bundle-branch block of premature ventricular beats, suggestive of early right ventricular activation via the myocardium rather than early left ventricular Purkinje system activation.

Mechanisms for ventricular arrhythmia formation during the delayed phase of ventricular arrhythmia development (6 to 12 minutes) after left circumflex coronary artery occlusion. Delayed ventricular arrhythmia formation was preceded by decreases in the prolonged activation delays observed on the epicardial surface after acute occlusion. As the epicardial delays partially returned to preocclusion values, there was a decrease in both the incidence and severity of ventricular arrhythmias. During the quiescent period, there was a continued increase in mid-myocardial activation delays in the circumflex coronary artery distribution. As shown in Figs. 3 to 5 , the mid-myocardial delays often exceeded the delays measured at the epicardial surface. The mid-myocardial delays could not result simply from the progressive slowing of conduction from the endocardial to epicardial surface. The premature ventricular beats were frequently $(23 \% \pm 8 \%)$ preceded by continuous diastolic electrical activation within acutely ischemic mid-myocardium. Even when continuous activity was absent during the delayed phase of ventricular arrhythmia development, the earliest site of activation on either composite or closely-spaced bipolar electrograms occurred within acutely ischemic mid-myocardium. His-bundle activation preceded the $\mathrm{QRS}$ interval. The frequent although not invariable presence of continuous electrical activity in mid-myocardium, the progressive increase in mid-myocardial activation delays that preceded the delayed phase of ventricular arrhythmia, the appearance of local conduction block in mid-myocardium preceding delayed ventricular arrhythmias (not observed on the epicardial surface), and the relatively early activation of the His-Purkinje system are suggestive of mid-myocardial reentry as a mechanism for the delayed phase of ventricular arrhythmia in the present experimental model.

Although two phases of ventricular arrhythmia have been previously described for acute left anterior descending coronary artery ligation, little information is available concerning the mechanism for delayed ventricular arrhythmia development. Kaplinsky et al. $^{3,11}$ have demonstrated that delayed ventricular arrhythmia occurs without antecedentdelayed epicardial electrical activity and antecedent epicardial conduction block or epicardial bridging of the diastolic interval preceding an ectopic ventricular beat. However, unlike the present study, delayed ventricular arrhythmias were not associated with progressive activation delays, local conduction block, or diastolic bridging in mid-myocardium. ${ }^{11}$ The observed differences could partly be explained by the use of summed or composite electrode recordings in the present study vs the closely spaced, individual bipolar electrode pairs used by Kaplinsky et al. ${ }^{11}$ The composite electrode recordings consisted of five bipolar pairs, each with an exposed recording surface of 2 to $3 \mathrm{~cm}$. Therefore electrical activation could be monitored throughout a large volume of acutely ischemic mid-myocardium. The earlier manifestation of the delayed phase of arrhythmia formation in the present study could have resulted from differences in the experimental model, that is, acute occlusion of the left circumflex coronary artery vs the left anterior descending coronary artery, or the presence of anterior infarction, sinus tachycardia, or an increase in sympathetic nervous system tone. $^{5}$

The role of the sympathetic nervous sytem in early ventricular arrhythmia formation after coronary artery occlusion. Acute myocardial ischemia activates sensory afferent sympathetic fibers within ischemic myocardium. ${ }^{12.14}$ Subsequent activation of the cardiocardiac sympathetic reflex produces dramatic increases in heart rate and myocardial contractility ${ }^{{ }^{15}}$ When the left circumflex coronary artery is occluded in the presence of previous anterior infarction, there is (1) an immediate and profound increase in the sinus heart rate ${ }^{5,6}$ and (2) the devel- 
opment of severe epicardial conduction delays in the left circumflex coronary artery distribution. ${ }^{6} \mathrm{~A}$ potential consequence of increased activation of the sympathetic nervous system is an increased incidence of ventricular fibrillation ( $90 \%$ to $95 \%)$ compared with proximal left circumflex coronary artery ligation in the absence of a previous ischemic event $(20 \%){ }^{6}$

Studies by Schwartz and Vanoli, ${ }^{16}$ Schwartz et al., ${ }^{17}$ and Euler et al. ${ }^{18}$ have demonstrated that electrical stimulation of the left stellate ganglion increases both the incidence and severity of ventricular arrhythmia observed during acute coronary artery ligation. The arrhythmogenic effect of left stellate stimulation occurs in both the presence ${ }^{16,17}$ and the absence ${ }^{18}$ of increased heart rates or arterial pressure. Pharmacologic and surgical interventions, which are capable of attenuating the effects of increased sympathetic nervous system tone (stellate ganglionectomy), ${ }^{5,19,20}$ surgical denervation, ${ }^{21,22}$ and $\beta$-adrenergic receptor blockade, ${ }^{11,23-25}$ lower the incidence of ventricular tachycardia and ventricular fibrillation accompanying acute coronary artery ligation.

Mechanism of action of $\beta$-adrenergic receptor blockade in the prevention of ventricular arrhythmia after coronary artery ligation. Despite strong evidence of a detrimental effect of increased sympathetic nervous system tone during acute myocardial ischemia, the bases for the beneficial electrophysiologic actions of $\beta$-adrenergic receptor blockade during myocardial ischemia are not well understood. Studies performed in anesthetized dogs by both Hope et al. ${ }^{10}$ and Reynolds et al. ${ }^{25}$ have suggested that reduction in the sinus heart rate, with concomitant reductions in epicardial conduction delays, ${ }^{10}$ is the primary component of the antiarrhythmic effect provided by propranolol. The protective effects can be substantially mitigated if heart rates are maintained at values ( 180 to 200 beats/min) observed in the control group. As in the present study, atrial pacing in both studies was maintained for the entire acute ischemic event. Therefore the possible detrimental effects of an acute or transient increase in heart rate on the ventricular rhythm cannot be separated from the adverse effects of the high heart rates on the ischemic process.

The present studies suggest that myocardial conduction delays exceeding 140 to $160 \mathrm{msec}$ are necessary but not sufficient for the initiation and maintenance of reentrant ventricular rhythms. Although a critical activation delay was achieved in the nadolol treatment group, the incidence and severity of arrhythmia after nadolol treatment were markedly reduced. Therefore the substantial reversal of the antiarrhythmic effect of $\beta$-adrenergic blockade with atrial pacing was accompanied not by significant changes in the overall magnitude of delayed myocardial activation observed in epicardium but by the absence of rate-dependent conduction disorders in ischemically injured mid-myocardium and epicardium.

It is possible that the protective effect of nadolol in the present experimental model could have been reversed by even transient periods of atrial pacing, which would have failed to alter the severity of the underlying ischemic process. The high heart rates, through an ability to expose rate-dependent conduction disorders or prolonged refractoriness within acutely ischemic ventricular myocardium, could act as a trigger to initiate reentry in an existing but otherwise quiescent substrate. A similar action of nadolol on the development of rapid ventricular heart beats triggering in an existing, quiescent substrate has been demonstrated for the 6- to 24-hour period after coronary artery ligation in the dog. ${ }^{26}$ The drug selectively suppressed the rapid, spontaneous ventricular beats associated with the spontaneous sustained ventricular tachycardia and fibrillation. The antiarrhythmic effect of $\beta$-adrenergic receptor blockade, that is, the failure to spontaneously initiate ventricular arrhythmia, was observed despite a failure to suppress the underlying reentrant substrate responsible for the lethal arrhythmic event.

Many of the electrophysiologic actions exerted by propranolol and other $\beta$-adrenergic receptor antagonists can be attributed to direct effects of the drug on myocardial conduction. ${ }^{27-29}$ In previous studies we have examined the electrophysiologic actions of nadolol in normal and ischemically injured tissues. ${ }^{7,8}$ At dosages of 0.125 to $8 \mathrm{mg} / \mathrm{kg}$, the drug fails to alter excitability thresholds, refractoriness, and conduction in either normal, acutely ischemic, or ischemically injured left ventricular myocardium. Studies conducted in vitro by Gibson et al. ${ }^{29}$ with intracellular microelectrode recordings have also failed to detect direct membrane actions of nadolol at concentrations exceeding those predicted by the present dosage regiment. Furthermore, in a previous study, ${ }^{7}$ we have demonstrated a failure of the $d$ nadolol, the inactive optical isomer of the racemic drug, to prevent ventricular fibrillation resulting from left circumflex thrombosis in the presence of previous anterior myocardial infarction. Therefore we must attribute the antiarrhythmic and antifibrillatory actions of $d, 1$-nadolol in the present study to $\beta$-adrenergic receptor blockade. 


\section{REFERENCES}

1. Waldo AL, Kaiser GA. A study of ventricular arrhythmias associated with acute myocardial infarction in the canine heart. Circulation 1973;47:1222.

2. Williams DO, Scherlag BJ, Hope RR, El-Sherif N, Lazzara R. The pathophysiology of malignant ventricular arrhythmias during acute myocardial ischemia. Circulation 1974;50:1163.

3. Kaplinsky E, Ogawa S, Balke CW, Dreifus LS. Two periods of early ventricular arrhythmia in the canine acute myocardial infarction model. Circulation 1979;60:397.

4. Boineau JP, Cox JT. Slow ventricular activation in acute myocardial infarction. Circulation 1973;48:702.

5. Schwartz PJ, Stone HL. Left stellectomy in the prevention of ventricular fibrillation caused by acute myocardial ischemia, in conscious dogs with anterior myocardial infarction. Circulation 1980;62:1256.

6. Patterson E, Holland K, Eller BT, Lucchesi BR. Ventricular fibrillation resulting from ischemia at a site remote from previous myocardial infarction: a conscious canine model of sudden coronary death. Am J Cardiol 1982;50:1414.

7. Patterson E, Lucchesi BR. Antifibrillatory actions of $d, 1$ nadolol in a conscious canine model of sudden coronary death. J Cardiovasc Pharmacol 1983;5:737.

8. Patterson E, Lucchesi BR. Antifibrillatory actions of nadolol. J Pharmacol Exp Ther 1982;223:144.

9. Lucchesi BR, Burmeister WE, Lomas TE, Abrams GB. Ischemic changes in the canine heart as affected by the dimethyl quaternary analog of propranolol, UM-272 (SC27761). J Pharmacol Exp Ther 1976;199:310.

10. Hope RR, Williams DO, El-Sherif N, Lazzara R, Scherlag BJ. The efficacy of antiarrhythmic agents during acute myocardial ischemia and the role of heart rate. Circulation 1974; 50:507.

11. Kaplinsky E, Ogawa S, Kmetza J, Balke CW, Dreifus LS. Intramyocardial activation in early ventricular arrhythmias following coronary artery ligation. J Electrocardiol 1980; 13:1.

12. Recordati G, Schwartz PJ, Malliani A, Brown AM. Activation of vagal receptors during myocardial ischemia. Experimentia $1971 ; 27: 1423$.

13. Thoren PN. Activation of left ventricular receptors with nonmedullated vagal afferent fibers during occlusion of a coronary artery in the cat. Am J Cardiol 1976;37:1046.

14. Malliani A, Recordati G, Schwartz. P.J. Nervous activity of afferent cardiac sympathetic fibers with atrial and ventricular endings. J Physiol (Lond) 1973;229:457.

15. Malliani A, Schwartz PJ, Zandetti A. A sympathetic reflex elicited by experimental coronary occlusion. Am J Physiol 1969;217:703.

16. Schwartz PJ, Vanoli E. Cardiac arrhythmias elicited by interaction between acute myocardial ischemia and sympathetic hyperactivity: a new experimental model for the study of antiarrhythmic drugs. I Cardiovasc Pharmacol 1981 3:1251.

17. Schwartz PJ, Vanoli E, Zaza A, Zuanetti G. The effect of antiarrhythmic drugs on life-threatening arrhythmias induced by the interaction between acute myocardial ische. mia and sympathetic hyperactivity. AM HEART J 1985;109:937.

18. Euler DE, Nattel S, Spear JF, Moore EN, Scanlon PJ. Effect of sympathetic tone on ventricular arrhythmias during circumflex coronary occlusion. Am J Physiol 1985;249:H1045.

19. Schwartz PJ, Stone HL, Brown AM. Effects of unilateral stellate ganglion blockade on the arrhythmias associated with coronary occlusion. AM HEART J 1976;92:589.

20. Fowlis RAF, Sang CTM, Lundy PM, Ahuja SP, Colhoun H. Experimental coronary artery ligation in conscious dogs six months after bilateral cardiac sympathectomy. AM HEART J 1974;88:748.

21. Schaal SF, Wallace AG, Sealy WC. Protective influence of cardiac denervation against arrhythmias of myocardial infarction. Cardiovasc Res 1969;3:241.

22. Ebert PA, Vanderbeek RB, Allgood RJ, Sabiston DC. Effect of chronic cardiac denervation on arrhythmias after coronary artery ligation. Cardiovasc Res 1970;4:141.

23. Fearon RE. Propranolol in the prevention of ventricular fibrillation due to experimental coronary artery occlusion. Am J Cardiol 1967;20:222.

24. Khan MI, Hamilton JT, Manning GW. Protective effect of beta-adrenergic blockade in experimental coronary artery occlusion in conscious dogs. Am J Cardiol 1972;30:832.

25. Reynolds RD, Calzadilla SV, Lee RJ. Spontaneous heart rate, propranolol, and ischemia-induced ventricular fibrillation in the dog. Cardiovasc Res 1978;12:653.

26. Patterson E, Scherlag BJ, Lazzara R. Mechanisms of prevention of sudden death by nadolol: differential actions on arrhythmia triggers and substrate after myocardial infarction in the dog. J Am Coll Cardiol 1986;8:1365.

27. Parmley WW, Braunwald E. Comparative myocardial depressant and antiarrhythmic properties of $d$-propranolol, d,1-propranolol, and quinidine. J Pharmacol Exp Ther 1967;158:11.

28. Lucchesi BR, Whitsett LS, Stickney JF. Antiarrhythmic effects of beta-adrenergic blocking agents. Ann NY Acad Sci 1967;139:940.

29. Gibson JK, Gelband H, Bassett AL. Direct and beta adrenergic blocking actions of nadolol (SQ 11725) on electrophysiologic properties of isolated canine myocardium. J Pharmacol Exp Ther 1977;202:702. 